\title{
Role and mechanism of micro-energy treatment in regenerative medicine
}

\author{
Yegang Chen ${ }^{1 \#}$, Qiliang Cai ${ }^{1 \#}$, Jiancheng Pan $^{1}$, Dingrong Zhang ${ }^{1}$, Jiang Wang ${ }^{1}$, Ruili Guan ${ }^{2}$, \\ Wenjie Tian $^{3}$, Hongen Lei ${ }^{4}$, Yuanjie Niu ${ }^{1}$, Yinglu Guo ${ }^{5}$, Changyi Quan ${ }^{1}$, Zhongcheng Xin ${ }^{1,2}$ \\ ${ }^{1}$ Department of Urology, the Second Hospital of Tianjin Medical University, Tianjin Institute of Urology, Tianjin 300211, China; ${ }^{2}$ Molecular \\ Biology Laboratory of Andrology Center, Peking University First Hospital, Peking University, Beijing 100034, China; ${ }^{3}$ Department of Urology, \\ Seoul St. Mary's Hospital, the Catholic University of Korea, Jongno-gu, Seoul, Korea; ${ }^{4}$ Department of Urology, Beijing Chao-Yang Hospital, Beijing \\ 100034, China; ${ }^{5}$ Department of Urology, Peking University First Hospital and the Institute of Urology, Peking University, Beijing 100034, China \\ Contributions: (I) Conception and design: Y Guo, Z Xin, C Quan; (II) Administrative support: C Quan, Z Xin, Y Niu; (III) Provision of study \\ materials or patients: Y Chen, Q Cai; (IV) Collection and assembly of data: J Pan, D Zhang; (V) Data analysis and interpretation: J Pan, D Zhang, J \\ Wang, R Guan, W Tian, H Lei; (VI) Manuscript writing: All authors; (VII) Final approval of manuscript: All authors. \\ \#These authors contributed equally to this work. \\ Correspondence to: Changyi Quan. Department of Urology, the Second Hospital of Tianjin Medical University, Tianjin Institute of Urology, Tianjin \\ 300211, China. Email: quancy98@163.com; Zhongcheng Xin. Department of Urology, the Second Hospital of Tianjin Medical University, Tianjin \\ Institute of Urology, Tianjin 300211, China; Molecular Biology Laboratory of Andrology Center, Peking University First Hospital, Peking \\ University, Beijing 100034, China. Email: xinzc@bjmu.edu.cn.
}

\begin{abstract}
With the continuous integration and intersection of life sciences, engineering and physics, the application for micro-energy in the basic and clinical research of regenerative medicine (RM) has made great progress. As a key target in the field of RM, stem cells have been widely used in the studies of regeneration. Recent studies have shown that micro-energy can regulate the biological behavior of stem cells to repair and regenerate injured organs and tissues by mechanical stimulation with appropriate intensity. Integrinsmediated related signaling pathways may play important roles in transducing mechanical force about microenergy. However, the complete mechanism of mechanical force transduction needs further research. The purpose of this article is to review the biological effect and mechanism of micro-energy treatment on stem cells, to provide reference for further research.
\end{abstract}

Keywords: Micro-energy medicine (MEM); stem cells; mechanical force; integrin; regeneration

Submitted Feb 08, 2020. Accepted for publication Feb 28, 2020.

doi: $10.21037 /$ tau.2020.02.25

View this article at: http://dx.doi.org/10.21037/tau.2020.02.25

\section{Introduction}

Micro-energy medicine (MEM) is an emerging integrated medicine that uses external devices to generate mechanical waves, electromagnetic waves and other forms of energy to prevent and treat diseases or sub-health states (1). In 2011, American Association for the Advancement of Science (AAAs) regarded integrated medicine as the "third revolution in life science" after cell and molecular biology and genomics, of which MEM is an important representative (2). Under concept of micro-energy, the core of life is no longer just the body, but energy. The intensity of body energy is directly reflected in cell viability. MEM can improve the vitality of cells in the body, through a variety of technical means, to achieve the goal of disease treatment and prevention (1). Recently, the application fields of MEM have been gradually extended to cardiology, urology, endocrinology, burn plastic surgery, oncology, even cosmetology. In addition to enhancing local blood circulation, stimulating peripheral nerve growth, antiinflammatory and analgesic effects, micro-energy treatment can also activate, transform and recruit stem cells to 
participate in the repair and regeneration of target organs and tissues (1). Compared with traditional treatments, micro-energy therapy, as a safer means, doesn't produce drug-related side effects and surgery-related complications.

Regenerative medicine ( $\mathrm{RM})$ is an emerging interdisciplinary field of life science and engineering, concentrated on repairing, replacing or regenerating injured tissues and organs to restore the damaged function caused by any reasons (3). The RM related technologies and methods include, but are not limited to, stem cell therapy, organ reconstruction in vitro, etc. (4). The applications of stem cell therapy in RM contain exogenous cell transplantation and endogenous cell activation (5). Stem cell transplantation has achieved remarkable results and been widely carried out, but there are still some problems after transplantation, such as the decline of cell survival rate and regeneration ability, immune rejection, ethical and tumorigenic problem. Therefore, it is difficult to generally and safely use stem cell banks for regenerative therapy in clinical (6). The body's innate healing response may also be leveraged to promote regeneration, although adult humans possess limited regenerative capacity. Many mammalian organs contain a subpopulation of undifferentiated stem cells (SC) involved in tissue replenishment and repair, e.g., the vascular endothelium can be repaired by the mature vessel wall-resident endothelial basal cells (stem cells) that migrate to the injured area. In a way, tissue resident stem cells are so-called endogenous stem cells. Owing to without immune rejection and ethical problem, Endogenous stem cells have attracted much attention in recent years (7). However, the problems, such as low numbers in vivo, slow cell proliferation and differentiation, are still existed.

Micro-energy therapy can promote the migration, proliferation and differentiation of stem cells from different sources, stimulate cells to secrete cell factors and regulate immune progress, to solve above problems. So, MEM has been widely used in the field of stem cell therapy and research. At present, the researches of the effect about MEM on the biological behavior of stem cells mostly stay at the phase of tissue and cell characterization after applying treatment. In order to further understand the regulation effect and mechanism of MEM on stem cells, this paper reviews the related research in recent years.

\section{Application classification of MEM}

The common treatment methods in MEM include lowintensity pulsed ultrasound (LIPUS), low-intensity extracorporeal shock waves therapy (Li-ESWT), and lowfrequency pulsed electromagnetic fields (PEMF).

\section{Li-ESWT}

Shock wave is a kind of bi-directional sound wave with frequency at $16 \mathrm{~Hz}-20 \mathrm{MHz}$, consisted by a short-time extremely high positive amplitude and a long-term low amplitude negative amplitude, transmitting energy to a remote anatomical target with minimal effect on the tissue located between the generator and the target (8). It has the characteristics of high peak pressure (up to $100 \mathrm{MPa}$ or higher), rapid pressure rise ( $<10 \mathrm{~ns})$ and short duration time $(<10 \mu s)(8)$, which can be divided into focused and divergent types. Shock wave disperses considerably less energy at $0.03-0.1 \mathrm{~mJ} / \mathrm{mm}^{2}$, is unfocused, and fewer shocks are administered. Li-ESWT, with usually less than that of $0.1 \mathrm{~mJ} / \mathrm{mm}^{2}\left(0.09 \mathrm{~mJ} / \mathrm{mm}^{2}\right.$ most common) energy flux density (EFD) (9), can produce mechanical force, micro-cavitation and thermodynamic effects to generate biological roles. Its energy density can be calculated as EFD $\times$ number of shock. Li-ESWT with $0.09 \mathrm{~mJ} / \mathrm{mm}^{2}$ shocked 50-400 times in each session can generate $4.5-36 \mathrm{~mJ} / \mathrm{mm}^{2}$ cumulative energy. Recently, Li-ESWT has been used in fracture healing, pain relief, arthritis treatment, soft tissue healing and revascularization, without causing damage at the site of action (10-13). Meanwhile, Li-ESWT also participates in the treatment of muscle diseases, heart disease, nonhealing or delayed healing wounds, and erectile dysfunction (ED) (14-17).

\section{Low frequency PEMF}

PEMF is a dynamic form of applied energy, which includes both magnetic and electric fields. Cells usually have a complex charge system, which is a potential target of electromagnetic action. PEMF can transfer energy and regulate the biological behavior of cells, which is achieved through the bioelectric fields produced by inductive coupling (18). PEMF, with fundamental (burst) frequency from 0 to $300 \mathrm{~Hz}$, the pulse frequency about $4 \mathrm{kHz}$, and magnetic field amplitude from some $\mathrm{mT}$ to several tens of $\mathrm{mT}$, has the characteristics of intermittent emission, square or trapezoidal waveform, and high safety $(19,20)$. As a micro-energy therapy method, PEMF can be used in bone healing, osteoporosis treatment and prevent $(21,22)$, and also act to improve angiogenesis, promote blood circulation and treat soft tissue injury, Parkinson's disease and other diseases (23-25). 


\section{LIPUS}

Ultrasound is a form of energy transfer for mechanical vibration, with a frequency from $2 \times 10^{4}$ to $2 \times 10^{9} \mathrm{~Hz}$ in transverse or longitudinal (26). When spreads in the organism, a certain dosage of ultrasound can cause changes of the organism function and structure, that is the biological effect of ultrasound. By adjusting the relevant parameters, scholars have found that ultrasound at the intensities of $0.05-0.50 \mathrm{~W} / \mathrm{cm}^{2}$ can be used in imaging medicine, and $0.03-1,000 \mathrm{~W} / \mathrm{cm}^{2}$ intensity ultrasound can be used for surgery and therapy (27).

As a widely used method in MEM, LIPUS emits lowintensity ultrasound $\left(<3 \mathrm{~W} / \mathrm{cm}^{2}\right)$ in the mode of pulse wave $(100-1,000 \mathrm{~Hz})$. The pulse period has $\mathrm{ON}$ and OFF signal periods, which are referred to as the duty cycle of the signal (26). LIPUS has good tissue penetration and low thermal effect, and realizes its therapeutic roles by mechanical effect. Most researchers used LIPUS with ultrasound intensity at $30 \mathrm{~mW} / \mathrm{cm}^{2}$, pulse ratio $1: 4$ at $1,000 \mathrm{~Hz}$, and frequency at $1.5 \mathrm{MHz}$ in their studies, and the cumulative energy (in $\mathrm{J} / \mathrm{cm}^{2}$ ) of LIPUS equals ultrasound intensity (in $\mathrm{W} / \mathrm{cm}^{2}$ ) times time (in seconds). Since the application time of LIPUS is about $2-20 \mathrm{~min}$, and the intensity range is from 0.03 to $1.00 \mathrm{~W} / \mathrm{cm}^{2}$, thus the commonly used energy density of LIPUS is about $2-150 \mathrm{~J} / \mathrm{cm}^{2}$ (27). In 1994 and 2000 respectively, the U.S. Food and Drug Administration (FDA) approved LIPUS as a safe and non-invasive method for the treatment of fresh fracture healing and nonunion (28). Moreover, LIPUS is also effective in promoting fibroblast differentiation, cartilage tissue, ligament, tendon repair and other treatments (29-33). Sports medicine research found that LIPUS is conducive to the penetration of topical drugs through the skin to injured site, reducing athlete recovery time (34).

\section{Biological effects of MEM on stem cells}

MEM could be capable of improving stem cell proliferation, self-renewal and other biological behaviors achieved by regulating cell cycle and promoting the expression of related genes $(35,36)$. LIPUS treatment significantly increased the expression of differentiation-related genes (such as osteocalcin) of mesenchymal stem cells and boosted their osteogenic differentiation. Meanwhile, endothelial differentiation inducer combined with LIPUS could more obviously advance the von Willebrand factor (vWF) expression and revascularization than didn't (37).
The results not only proved that LIPUS could improve stem cell differentiation, but also suggested that LIPUS combined with other stem cell differentiation inducing factors could increase the multi-directional differentiation ability of stem cells. MEM could also regulate the migration of mesenchymal stem cells to the treated site through rearranging cytoskeleton and expressing cell migrationrelated genes (such as ITGA8), in vivo and in vitro. Simultaneously, the recruited stem cells secreted a large number of cellular active factors, such as nerve growth factor (NGF) and vascular endothelial growth factor (EGF), to promote the damaged nerves and vessels repair $(38,39)$. Literature review found that in a certain range, the biological efficacy of MEM on stem cells increased with the increase of its dosage, but excessive energy beyond a threshold would lead to some negative results, for example, cell apoptosis $(40,41)$.

\section{Effect of MEM on exogenous stem cells}

Exogenous stem cells used for stem cell transplantation in RM usually have the cross-system, cross-derm differentiation potential and high self-renewal ability, which can be divided into two types: embryonic stem cells and adult stem cells. Embryonic stem cells have great medical potential as omnipotent stem cells, but they cannot be used for clinical treatment owing to the ethical problems. Therefore, researchers gradually focus on adult stem cells, including bone marrow mesenchymal stem cells (BMSCs), adipose-derived stem cells (ADSCs) and others.

\section{Effect of MEM on BMSCs}

BMSC, a kind of adult stem cell deriving from bone marrow, is more suitable for stem cell transplantation than other cells because of its advantages such as convenient material acquisition, low immune rejection and easy foreign gene transfection and expression. Therefore, BMSCs have been expected to be an ideal tool in the field of cell therapy in RM. However, some defects in BMSCs practical application are also existing, for instance low efficiency of proliferation and differentiation, low survival rate, and small number of cells homing to the target organs (42). Many works regarding BMSCs have demonstrated MEM could effectively improve above problems.

MEM-mediated BMSCs transplantation is most widely used in fracture researches. In a study, researchers found that in vitro LIPUS (unfocused plane waves, frequency $1.5 \mathrm{MHz}$, duty cycle $1: 4$, spatial average-temporal average 
intensity $30 \mathrm{~mW} / \mathrm{cm}^{2}$, pulse repetition frequency $1 \mathrm{kHz}$ for pulse duration of $200 \mu \mathrm{s}, 20$ minutes/day, 7 days) increased the stromal cell derived factor-1 (SDF-1) and C-X-C chemokine receptor type 4 (CXCR4) mRNA levels about 1.6 times and 4.3 times than control group without LIPUS treatment respectively and promoted migration of BMSCs in cell migration assay $(\mathrm{P}=0.003)$. Compared with injection of BMSCs alone in vivo, LIPUS combined with BMSCs transplantation significantly improved the earlier fracture healing and callus formation (43).

Moreover, Sansone et al. (44) proposed a novel approach for the treatment of atrophic bone nonunions via parallel applications of Li-ESWT and BMSCs transplantation. Li-ESWT could be employed to induce up-regulation and expression of several angiogenic and osteogenic growth factors, which promoted BMSCs replication and differentiation (44). Another research about PEMF and BMSCs demonstrated that PEMF $(20 \mathrm{~Hz} / 2 \mathrm{mT})$ significantly advanced the activity of the alkaline phosphatase $(\mathrm{ALP})(\mathrm{P}<0.01)$ and the expression of osteocalcin (osteocalcin and osteopontin) in BMSC, while inhibited adipogenic differentiation of BMSCs by reducing adipogenic factor expression (45). In addition, icariin as a means of regulating osteogenic differentiation of BMSCs has been reported (46), so whether icariin combined with MEM can enhance the regulatory effect of BMSCs is worthy of further study.

MEM combined with BMSCs also plays a role in treating spinal cord, kidney, heart injury and diabetic ED. Ning et al. (47) found that BMSCs stimulated by LIPUS (10, 30, $50,70 \mathrm{~mW} / \mathrm{cm}^{2}, 3 \mathrm{~min} / \mathrm{d}, 3$ days) had higher cell viability in cell proliferation, especially cells in $50 \mathrm{~mW} / \mathrm{cm}^{2}$ group. It can be seen that LIPUS has the optimum intensity for stem cell action. Meanwhile, LIPUS treatment enhanced BMSCs migration ability $(\mathrm{P}<0.0001)$ and cell neurotrophic factors expression $(\mathrm{P}<0.0001)$ than control in vitro. BMSCs stimulated by LIPUS could increase the content of brainderived neurotrophic factor $(\mathrm{BDNF})$ and $\mathrm{NGF}(\mathrm{P}<0.0001)$, reducing reactive gliosis and recovering rat locomotor function. Furthermore, the cavity formation of injured spinal cord rates in LIPUS-treated BMSCs group reduced $20 \%$ than BMSCs transplantation group $(\mathrm{P}<0.01)(47)$. The recent research suggested that LIPUS [frequency $1 \mathrm{MHz}$, duty cycle $10 \%$, peak negative pressure $0.35 \mathrm{MPa}$ $\left(0.6 \mathrm{~W} / \mathrm{cm}^{2}\right)$, micro-bubble dosage $10^{6} / \mathrm{mL}$, and total ultrasound sonication time $30 \mathrm{~s}$ ] combined with microbubbles could promote CXCR-4 modified BMSCs homing to acute renal injury tissue of rat $(\mathrm{P}<0.01)$, participating in tissue repair, and recovering renal structure and function (48). Chang and other scholars (49) conducted a control study about the effect of the dog model of myocardial infarction treated by BMSCs transplantation, received LIPUS along with microbubbles treatment or not. Results demonstrated that the pretreated BMSCs more gathered in the coronary artery, especially in the infarcted area, and the group of BMSCs transplantation received ultrasound-mediated microbubble destruction showed the most improvement in heart function and percentage of perfusion defect area to total left ventricular perfusion area $(\mathrm{P}<0.05)$. In 2017, Shan et al. (50) found that combination of Li-ESWT and BMSC improved erectile function of diabetic erectile function rats more effectively than BMSC transplantation alone. The reason might be related to that $\mathrm{Li}$ ESWT favored the survival of transplanted BMSCs, increased stromal cell-derived factor-1 expression and enhanced revascularization in the diabetic cavernous tissue (50).

\section{Effect of MEM on ADSCs}

Compared with BMSCs, the ADSCs' proliferation and differentiation potential have no advantage. However, ADSCs sources are richer and easier to obtain, so that the researches on ADSCs are increasing in recent years. There are also still difficulties in ADSCs transplantation, and the roles of MEM on ADSCs transplantation have been widely reported. Jiang et al. (51) found that ADSCs treated by LIPUS (frequency 1.0 MHz, pulse repetition frequency $1 \mathrm{kHz}$ for pulse duration of $200 \mu \mathrm{s}$, at $100 \mathrm{~mW} / \mathrm{cm}^{2}$ ) displayed higher mRNA expression levels of runt-related transcription factor 2 (Runx2), osteocalcin (OCN), ALP and bone sialoprotein (BSP) genes than ADSCs injection alone $(\mathrm{P}<0.05)$, which suggested LIPUS can induce the osteogenic differentiation of ADSCs (51). Another study proved ADSCs pretreated by Li-EWST had potent therapeutic effect in diabetic bladder dysfunction by secreting VEGF and NGF to improve bladder innervation and revascularization. Compared with untreated ADSCs injection, injecting treated ADSCs also enhanced the migrations of endogenous stem cells to injured sites $(\mathrm{P}<0.05)(52)$. The roles of PEMF in regulating the osteogenic ability of human adipose-derived mesenchymal stromal cells (hADSC) have been also reported (53). Nasb et al. (54) conducted the first clinical study about LIPUS on ADSCs therapy in OA patients. Patients were randomly divided into 3 groups in a ratio of 1:1:1, respectively treated by injecting ASDC, injecting saline with LIPUS treatment (pulsed frequency 
$1 \mathrm{MHz}$ and $0.5 \mathrm{w} / \mathrm{cm}^{2}$ intensity, $20 \mathrm{~min} /$ day, 5 days/week for 8 weeks), and injecting ASDC with the same LIPUS treatment (same above). But the results have not been obtained. Many ways can enhance the efficacy of ADSCs transplantation in the treatment of ED. As one of the above methods, icariin can greatly improve the survival rate of transplanted ADSCs, and increase the expression of $\mathrm{vWF}$ and $\alpha$-SMA (54). In addition, another study also found that icariin II could also repair penile nerve by inducing ADSCs to differentiate into Schwann cells (SCs), and thus enhance erectile function (55). However, the research of MEM along with ADSCs for ED therapy is rare.

\section{Effect of MEM on other stem cells}

There are some studies on the relation between other stem cells transplantation therapy and MEM. Ling and other scholars (56) isolated human amnion-derived mesenchymal stem cell (hAD-MSC), treated them with LIPUS (frequency $0.25 \mathrm{MHz}$, burst width sine wave $200 \mu$ s, duty cycle $20 \%$, pulse repetition frequency, $1 \mathrm{kHz}$ ), and then injected them into the caudal vein of rats with ovarian insufficiency. Results revealed that LIPUS promoted hAD-MSCs to express and secret growth factors in vitro. And LIPUSpretreated hAD-MSC transplantation increased the weight of reproductive organs, improved ovarian function, and reduced ovarian inflammation than untreated hAD-MSC transplantation (all $\mathrm{P}<0.01)(56)$. As a class of stem cells with multi-directional differentiation potential obtained from somatic cell reprogramming, induced pluripotent stem cells (iPSCs) have less immunogenicity and do not involve ethical issues. Xia et al. (57) conducted a randomized, shamcontrolled study using LIPUS and iPSCs for nerve injured rat. LIPUS combined with iPSCs injection enhanced the repair of transected sciatic nerve in rats than iPSCs transplantation and LIPUS treatment alone $(\mathrm{P}<0.05)$. Meanwhile, this study demonstrated that LIPUS obviously promoted the neural differentiation of iPSCs in vitro (57). But iPSCs may have potential problems because of its modified genes. Therefore, longer-term observation and research for the safety of iPSCs therapy are also needed.

\section{Effect of MEM on endogenous stem cells}

MEM combined with exogenous stem cell transplantation has achieved many good results in basic and animal experiments for a variety of diseases, repaired the injured tissues and significantly improved their functions. However, due to the existence of immunogenicity, tumorigenic risk and potential ethical problems, the clinical transformation of exogenous stem cell transplantation is difficult. Compared with exogenous stem cell transplantation treatment, individual endogenous stem cell therapy can effectively avoid above risks. Therefore, scholars turn their attention into the treatment of endogenous stem cells, but there are also still some problems, such as small number of endogenous stem cells, lag of cell activation and slow differentiation process. These are the reasons why the studies on the effect of MEM on endogenous stem cells are increasing as time goes on.

\section{Effect of MEM on myogenic stem cells}

The relatively unified structure and the simple cells composition of muscle tissue make it easier to track the biological behavior of myogenic stem cells. At the same time, endogenous muscle-derived stem cells are safe on muscle tissue repair, and also their behaviors could be regulated by micro-energy treatment.

According to recent study, Yang et al. (58) used LIPUS [intensity $0,100,200$, and $300 \mathrm{~mW} / \mathrm{cm}^{2}$, pulse on and off time ratio of 1:4 (200 $\mu \mathrm{s}: 800 \mu \mathrm{s})$ at $1,000 \mathrm{~Hz}$ and frequency at $1.7 \mathrm{MHz}, 3$ minutes each time, every 2 days for 7 times] to treat rats of stress urinary incontinence (SUI). The results demonstrated that in $200 \mathrm{~mW} / \mathrm{cm}^{2}$ and $300 \mathrm{~mW} / \mathrm{cm}^{2}$ groups, the bladder capacity (BC) and leak point pressure (LPP) were restored to a normal level, and $200 \mathrm{~mW} / \mathrm{cm}^{2}$ LIPUS promote the regeneration of striated muscle $(\mathrm{P}<0.05)$, that plays important role in urinary continence function. Furthermore, $200 \mathrm{~mW} / \mathrm{cm}^{2}$ LIPUS could also enhance activation of satellite cell myogenic differentiation $(\mathrm{P}<0.05)$ (58). In 2018, Wu et al. (59) found that Li-ESWT $\left(0.06 \mathrm{~mJ} / \mathrm{mm}^{2}\right.$, and 300 shocks at $\left.3 \mathrm{~Hz}\right)$ improved urethral muscle regeneration of SUI rats model $(\mathrm{P}<0.05)$, and significantly increased EdU+ endogenous progenitor cells in the muscle layer, particularly the striated muscle. Moreover, Li-ESWT could also promote rat myoblast to format myotube in vitro (59). Kang et al. (60) also proposed that compared with untreated Zucker Fatty rats (a rat model of obese-related SUI), micro-energy acoustic pulses (at $0.033 \mathrm{~mJ} / \mathrm{mm}^{2}, 3 \mathrm{~Hz}$ for 500 pulses, two times a week for 2 weeks) significantly increased $\mathrm{H} 3 \mathrm{P}+$ cells in pelvic floor muscle and urethra of $\mathrm{ZF}(\mathrm{P}<0.01)$. Furthermore, microenergy acoustic pulses promote stem cells differentiation into satellite cells in rat pelvic layer muscle and urethra (60). These results indicate that MEM, act to endogenous stem cells, represents a potential novel non-invasive therapy for SUI. 
In the aspect of ED therapy, in 2016, Behr-Roussel et al. (61) had made the assumption that Li-ESWT could trigger the activation of various intracellular signaling pathways causing up-regulation of numerous angiogenic factors (i.e., VEGF) to recruit stem cells and promote them differentiation to form neovascularization, and then improve erectile function. Moreover, in 2019, scholars identified and localized the penile stem/progenitor cells (PSPCs) with multiple differentiation characterize. Meanwhile, they found that Li-EWST $\left(0.033 \mathrm{~mJ} / \mathrm{mm}^{2}, 1 \mathrm{~Hz}, 50\right.$ pulses $)$ enhanced expression of $\alpha$-SMA from $0.14 \pm 0.01$ to $0.31 \pm 0.02(\mathrm{P}<0.001)$ of PSPCs, and provoked cells to differentiate into smooth muscle cells rather than skeleton muscle cells in vitro $(62,63)$. Both animal and cell experiments have verified the positive effect of Li-ESWT treatment for ED by acting endogenous stem cells. In addition, almost ED patients experienced improvements in their erectile condition following $\mathrm{Li}-$ ESWT therapy (64), which supported the above conclusion.

\section{Effect of MEM on neural stem cells}

The repair of spinal cord and nerve injury can be achieved by activating neural stem cells. The biological effects of MEM on neural stem cells have also been reported. Compared with the other mechanical waves, the PEMF is more penetrating and safer in the neural tissue.

Hei et al. (65) performed a sham-control trail using PEMF (1 mT, different frequency) for SCs. the result demonstrated that in condition of $50 \mathrm{~Hz}, 1 \mathrm{~h} / \mathrm{d}$ SCs was increased from 4th-7th day, and mRNA expression of $\mathrm{BDNF}$ and S100 was significantly increased at the same condition from first week to third week $(\mathrm{P}<0.05)$ than other conditions of PEMF treatment. In addition, researchers also used PEMF ( $1 \mathrm{mT}$, different frequency) to treat never injured rats, and then counted axon and neurons labeled with Dil in damaged never. They found that PEMF with different frequency $(50 \mathrm{~Hz} 1 \mathrm{~h} /$ day, $50 \mathrm{~Hz} 12 \mathrm{~h} /$ day, $150 \mathrm{~Hz} 1 \mathrm{~h} /$ day and $150 \mathrm{~Hz} 12 \mathrm{~h} /$ day) could also significantly regenerate axon and neurons, and in $50 \mathrm{~Hz}$ $1 \mathrm{Hr} /$ day condition, the regeneration was most obvious. The results of animal and cell studies are consistent in the best suitable frequency (65). Another study, Vincenzi et al. (66) found that the contents of hypoxia-inducible factor-1 (HIF-1), reactive oxygen species (ROS) and inflammatory cytokines in rat injured nerves were decreased by PEMF, which was greatly reducing neuron-Like and microglial cells death and apoptosis.

The protective effect on hypoxic injury of neural stem cells and the role of promoting nerve regeneration lead
PEMF to a potential treatment for cerebral ischemia and other nerve injury diseases.

\section{Mechanism of MEM action on stem cells}

Ultrasound and shock waves can generate thermal energy through mechanical wave vibration, while electromagnetic waves use non-ionizing radiation to cause local temperature rise. Although suitable thermal energy can regulate the expression of cell-related factors and biological behaviors, such as cell metabolism, these effects are too weak. Thus, thermal effect is not discussed as the main mechanism.

Many studies have considered mechanical effects as the most important biological effects of ultrasound and shock waves on stem cells. Stem cells recognize mechanical signals through certain cell membrane proteins or cell structures, and respond to activate related protein pathways within the cell, causing changes in function of key proteins and gene expression. The process of receiving mechanical signals, activating cellular signaling pathways, and conduction between different cells can be divided into three phases, including: mechanical coupling phase, biochemical coupling phase, and signal transmission phase (67).

How cells transduce the mechanical force into biochemical stimulation is not completely understood. At present, it is commonly believed that the cell structures participating in mechanical force transduction include integrins, ion channels, specific cytoskeleton proteins, cell junction molecules, and certain mechanical sensitivity protein complexes. In these structures, integrins, mechanosensitive ion channels are studied more widely.

In a study of mechanical signal activating mechanosensitive $\mathrm{Ca}^{2+}$ channels, it was found that the stimulation of mechanical force with appropriate intensity caused cytoskeleton proteins to pull ion channels, leading to channels open and $\mathrm{Ca}^{2+}$ influx (68). Li-ESWT can also stimulate connexin 43 hemichannels to release ATPs, which activate $\mathrm{P} 2 \mathrm{Y} 1$ purinergic receptors and then trigger the PLC-IP3-IP3R cascade to release $\mathrm{Ca}^{2+}$ into the cytoplasm (69). Moreover, Gao et al. (70) found that dental pulp stem cells (DPSC) presented membrane ion channels Piezo1, participating in DPSC proliferation mediated by LIPUS. In 2018, Zhao et al. (71) revealed that piezo1 channel is a propeller-shaped three-bladed trimeric architecture, consisted by central ion-conducting pore and the peripheral blade-like structures. When piezo1 was stimulated by mechanical force, its peripheral blade-like structures and the distal parts of beam changed 
dramatically, while the central pore area and the central part of beam showed only slight displacement, and these piezo1 structural changes conformed to the principle of leverage. Combined with previous researches, they proposed the piezo1 mechanosensitive mechanism hypothesis that transmembrane helical unit (THU), the structural units of peripheral blade, and beam were regarded as the mechanical force sensor and mechanical transmission device respectively, to regulate channels open or close, which caused changes about intracellular cation concentration, especially $\mathrm{Ca}^{2+}$, to regulate cell behaviors (71). Increase of $\mathrm{Ca}^{2+}$ within cells can affect the synthesis of cell growth factors and gene expression by regulating $\mathrm{Ca}^{2+}-$ dependent enzyme activity and activating downstream signaling pathways (69). Other mechanosensitive ion channels include MscL, YAP, etc. (72,73). Pilla (74) found that electromagnetic fields can also activate cation channels, to further activate the second messenger and affect gene transcription, eventually realizing the role of PEMF in regulating cell activity.

Recently, studies demonstrated that ultrasound effect on stem cells to cause cell proliferation and differentiation is closely related to the integrin in the cell membrane (75), and Li-ESWT can also induce stem cell differentiation mediated by integrin (76). In the inactivated state, the integrin conformation is usually in a folded condition and exhibits low affinity for ligand binding. When cells encounter certain activation signals, related regulatory proteins in the cell, such as talin and kindlin proteins, are induced to bind to cytoskeletal protein and the intracellular region of integrin, inducing integrin conformation to change from a folded state to an extended state, and hence increasing ligand-biding affinity $(77,78)$. The ligand (such as fibrin, laminin, glassin) binding to integrin can also further induce conformational changes and clustering of integrin on the cell membrane surface (79), thereby activating multiple protein kinases and phosphatases binding to integrin intracellular regions. These macromolecular protein complexes contain focal adhesion kinase (FAK), Src kinase, integrin linked kinase (ILK), etc. Most studies focused on FAK and FAK-mediated downstream pathways, including FAK-Ras-MAPK, FAK-PI3K-AKT/PKB, FAK-STAT1, FAK-GTPase, etc. (80). FAK conformation changes lead to its autophosphorylation, which make downstream MAP3Ks, Ras phosphorylation, and then activate MAPK and PI3KAKT pathways. As one intracellular signaling pathway participating in differentiation of myogenic cell, activated P38 MAPK promotes the activity of transcription factors of the MyoD families and takes part in the remodeling of muscle-regulatory related chromatin (81), and also phosphorylates $\mathrm{E} 47$, promoting its heterodimerization with MyoD. The MyoD-E47 heterodimerization activates the muscle-specific transcription (82). Furthermore, $\mathrm{PI} 3 \mathrm{~K} / \mathrm{AKT}$ signalling pathway plays a role in the control of stem cells pluripotency and differentiation. Active PI3K phosphorylates phosphatidylinositol-4,5-bisphosphate (PIP2) to phosphatidylinositol-3,4,5-triphosphate (PIP3), and then recruits and phosphorylates AKT, as well as activates mTORC2 (83). Activated AKT can improve the activation of mTORC1, which regulates cell cycle-regulated protein expression and promotes cell cycle conversion from G0 to G(Alert). The transition of quiescent stem cells into G(Alert) functions positions stem cells to respond rapidly under conditions of injury and stress, priming them for cell cycle entry (84). Meanwhile, mTORC1 can also increase stem cell activity and promote cell proliferation (85). In addition, mTORC2 maintains cell survival and metabolism (86). Other identified pathways activated by micro-energy treatment include: JNK, Wnt, PERK/ATF, ATP/P2X7, Notch (87). And many important pathways have yet to be discovered. The processes of Micro-energy mechanical force transduction mediated by integrin are as shown in Figure 1, including three maintain signaling pathways.

Ultrasound (or shock wave) microbubbles, exceed a certain threshold, can cause a sonoporation effect, that is, the above methods make the cell membrane surface temporarily disruption, which promote extracellular molecules to enter the cell, causing changes in cell biological behaviors (88). This effect is widely used in experimental studies such as cell transfection and drug infusion, and also provides a novel assumption than traditional opinion of mechanical force transduction.

In order to improve the therapeutic theory of MEM, it is of great significance to clarify the mechanism of action of MEM on cells and completely understand the specific ways and regulatory process of the effect.

\section{Limitation}

Although increasing scholars affirm the effects of MEM, there are more and more worries. MEM can regulate cell functions through different mechanisms such as mechanical effects and electromagnetic effects, but there is no uniform standard for the indexes. Currently, there are a variety of MEM instruments used for basic research or clinical 


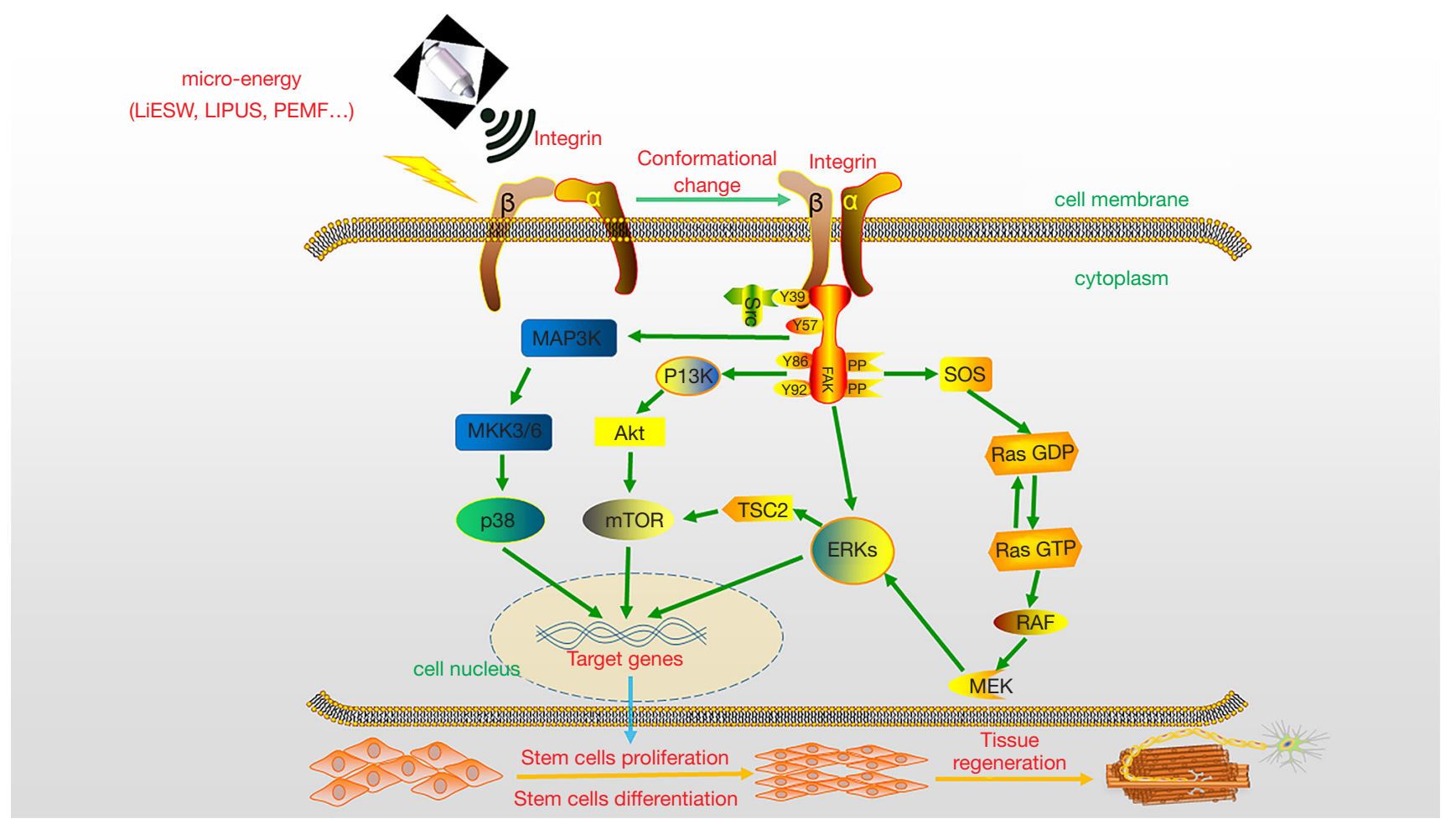

Figure 1 Important role and mechanism of micro-energy treatment in regenerative medicine. LIPUS, low-intensity pulsed ultrasound; PI3K, phosphoinositide 3-kinase; Akt, protein kinase B (PKB), also known as Akt; Src, Src is short for sarcoma, a proto-oncogene tyrosineprotein kinase Src; TSC2, tuberous sclerosis complex 2; RAS, molecules of MAPK/ERK pathway; RAF, molecules of MAPK/ERK pathway; MEK, mitogen-activated protein kinase kinase; mTOR, mammalian target of rapamycin complex 1; FAK, focal adhesion kinase; SOS, Son of Sevenless; MAP3K, MAP kinase kinase kinase; MKK3/6, MAP kinase kinase 3/6; p38, p38 MAPK.

therapy, such as the MTS Dermagold (Atlanta, GA, USA), WBL-ED (Beijing, china), TY-PEMF-CS (Shanghai, china) and others, and also their parameters criterion and ranges are not coincident. The FDA stipulated the safety range of diagnostic ultrasound, including mechanical effect index, thermodynamic effect index and exposure time (89), but the non-imaging medicine safety standard for MEM has not been proposed. The cells with different types have different response and tolerance for micro-energy treatment, and the difference is more significantly in animal experiments. Human tissue structure is more complex, so parameters setting is more difficult. Improper stimulation would damage tissues and cells, even lead to more fearful potential consequences, so it is notably important to understand the mechanism of MEM on cells.

\section{Summary}

The related studies on the biological effects of MEM on stem cells have proved that MEM is a safe and effective treatment in RM. Meanwhile, due to the numerous insurmountable risks of exogenous stem cell transplantation, endogenous stem cell micro-energy might be important target of micro-energy in regeneration. Micro-energy regulates stem cells mainly through its mechanical effects. Stem cells recognize and transduce cell surface mechanical force signals to intracellular biochemical signals through specialized cellular structures, and further activating a large number of downstream signaling pathways to achieve the regulation of cell biological behaviors, such as proliferation and differentiation. Integrins as important cell membrane mechanosensitive proteins play key roles in the above micro-energy mechanical force transduction process. At the same time, other possible mechanical force transduction ways and intracellular related downstream signaling pathways have been deeper and more extensive in recent years Thus, existing researches make the mechanisms by which micro-energy treatment regulates the involvement 
of stem cells in tissue and organ regeneration clearer, and also propose possible directions and challenges for future research. In conclusion, the integration of life sciences, physics and engineering will significantly promote the advancement of MEM in RM and bring good news for human health.

\section{Acknowledgments}

Funding: This study was funded by Natural Science Foundation of Tianjin (No. 17JCQNJC11900) and Tianjin Technical Expert Project.

\section{Footnote}

Conflicts of Interest: All authors have completed the ICMJE uniform disclosure form (available at http://dx.doi. org/10.21037/tau.2020.02.25). The authors have no conflicts of interest to declare.

Ethical Statement: The authors are accountable for all aspects of the work in ensuring that questions related to the accuracy or integrity of any part of the work are appropriately investigated and resolved.

Open Access Statement: This is an Open Access article distributed in accordance with the Creative Commons Attribution-NonCommercial-NoDerivs 4.0 International License (CC BY-NC-ND 4.0), which permits the noncommercial replication and distribution of the article with the strict proviso that no changes or edits are made and the original work is properly cited (including links to both the formal publication through the relevant DOI and the license). See: https://creativecommons.org/licenses/by-ncnd/4.0/.

\section{References}

1. Guo Y, Xin Z, Li H, et al. Welcome the third revolution of life science to attach importance for the development of micro-energy medicine. Journal of Peking University (Medical Edition) 2015;47:559-65.

2. Sharp PA, Langer R. Research agenda. Promoting convergence in biomedical science. Science 2011;333:527.

3. Mao AS, Mooney DJ. Regenerative medicine: Current therapies and future directions. Proc Natl Acad Sci U S A 2015;112:14452-9.

4. Jacques E, Suuronen EJ. The Progression of Regenerative
Medicine and its Impact on Therapy Translation. Clin Transl Sci 2020. [Epub ahead of print].

5. Trohatou O, Roubelakis MG. Mesenchymal Stem/Stromal Cells in Regenerative Medicine: Past, Present, and Future. Cell Reprogram 2017;19:217-24.

6. Rota C, Morigi M, Imberti B. Stem Cell Therapies in Kidney Diseases: Progress and Challenges. Int J Mol Sci 2019. doi: 10.3390/ijms20112790.

7. Stenudd M, Sabelström H, Frisén J. Role of endogenous neural stem cells in spinal cord injury and repair. JAMA Neurol 2015;72:235-7.

8. Wang HJ, Cheng JH, Chuang YC. Potential applications of low-energy shock waves in functional urology. Int J Urol 2017;24:573-81.

9. d'Agostino MC, Craig K, Tibalt E, et al. Shock wave as biological therapeutic tool: From mechanical stimulation to recovery and healing, through mechanotransduction. Int J Surg 2015;24:147-53.

10. Xu JK, Chen HJ, Li XD, et al. Optimal intensity shock wave promotes the adhesion and migration of rat osteoblasts via integrin beta1-mediated expression of phosphorylated focal adhesion kinase. J Biol Chem 2012;287:26200-12.

11. Kertzman P, Csaszar NBM, Furia JP, et al. Radial extracorporeal shock wave therapy is efficient and safe in the treatment of fracture nonunions of superficial bones: a retrospective case series. J Orthop Surg Res 2017;12:164.

12. Li W, Pan Y, Yang Q, et al. Extracorporeal shockwave therapy for the treatment of knee osteoarthritis: a retrospective study. Medicine (Baltimore) 2018;97:e11418.

13. Rassweiler JJ, Knoll T, Kohrmann KU, et al. Shock wave technology and application: an update. Eur Urol 2011;59:784-96.

14. Hazan-Molina H, Reznick AZ, Kaufman H, et al. Periodontal cytokines profile under orthodontic force and extra-corporeal shock wave stimuli in a rat model. J Periodontal Res 2015;50:389-96.

15. Yang P, Guo T, Wang W, et al. Randomized and doubleblind controlled clinical trial of extracorporeal cardiac shock wave therapy for coronary heart disease. Heart Vessels 2013;28:284-91.

16. Cooper B, Bachoo P. Extracorporeal shock wave therapy for the healing and management of venous leg ulcers. Cochrane Database Syst Rev 2018;6:CD011842.

17. Fojecki GL, Tiessen S, Osther PJ. Extracorporeal shock wave therapy (ESWT) in urology: a systematic review of outcome in Peyronie's disease, erectile dysfunction and chronic pelvic pain. World J Urol 2017;35:1-9. 
18. Yuan J, Xin F, Jiang W. Underlying Signaling Pathways and Therapeutic Applications of Pulsed Electromagnetic Fields in Bone Repair. Cell Physiol Biochem 2018;46:1581-94.

19. Waldorff EI, Zhang N, Ryaby JT. Pulsed electromagnetic field applications: A corporate perspective. J Orthop Translat 2017;9:60-8.

20. Hug K, Röösli M. Therapeutic Effects of Whole-Body Devices Applying Pulsed Electromagnetic Fields (PEMF): A Systematic Literature Review. Bioelectromagnetics 2012;33:95-105.

21. Pilla A. Pulsed Electromagnetic Fields: From Signaling to Healing. Electromagnetic Fields in Biology and Medicine. Boca Raton, FL: CRC Press, 2015:29-48.

22. Selvamurugan $\mathrm{N}, \mathrm{He} Z$, Rifkin $\mathrm{D}$, et al. Pulsed electromagnetic field regulates microRNA 21 expression to activate TGF-b signaling in human bone marrow stromal cells to enhance osteoblast differentiation. Stem Cells Int 2017;2017:2450327.

23. Cameron IL, Markov MS, Hardman WE. Optimization of a therapeutic electromagnetic field (EMF) to retard breast cancer tumor growth and vascularity. Cancer Cell Int 2014;14:125.

24. Pesce M, Patruno A, Speranza L, et al. Extremely low frequency electromagnetic field and wound healing: implication of cytokines as biological mediators. Eur Cytokine Netw 2013;24:1-10.

25. Vadalà $M$, Vallelunga A, Palmieri L, et al. Mechanisms and therapeutic applications of electromagnetic therapy in Parkinson's disease. Behav Brain Funct 2015;11:26.

26. Harrison A, Lin S, Pounder N, et al. Mode \& mechanism of low intensity pulsed ultrasound (LIPUS) in fracture repair. Ultrasonics 2016;70:45-52.

27. Xin Z, Lin G, Lei H, et al. Clinical applications of lowintensity pulsed ultrasound and its potential role in urology. Transl Androl Urol 2016;5:255-66.

28. Ebrahim S, Mollon B, Bance S, et al. Low-intensity pulsed ultrasonography versus electrical stimulation for fracture healing: a systematic review and network meta-analysis. Can J Surg 2014;57:E105-18.

29. Zhou S, Schmelz A, Seufferlein T, et al. Molecular mechanisms of low intensity pulsed ultrasound in human skin fibroblasts. J Biol Chem 2004;279:54463-9.

30. Cook SD, Salkeld SL, Patron LP, et al. The effect of lowintensity pulsed ultrasound on autologous osteochondral plugs in a canine model. Am J Sports Med 2008;36:1733-41.

31. Ikai H, Tamura T, Watanabe T, et al. Low-intensity pulsed ultrasound accelerates periodontal wound healing after flap surgery. J Periodontal Res 2008;43:212-6.

32. Sparrow KJ, Finucane SD, Owen JR, et al. The effects of low intensity ultrasound on medial collateral ligament healing in the rabbit model. Am J Sports Med 2005;33:1048-56.

33. Hu J, Qu J, Xu D, et al. Combined application of lowintensity pulsed ultrasound and functional electrical stimulation accelerates bonetendon junction healing in a rabbit model. J Orthop Res 2014;32:204-9.

34. Tsukada M, Takiuchi T, Watanabe K. Low-Intensity Pulsed Ultrasound for Early-Stage Lumbar Spondylolysis in Young Athletes. Clin J Sport Med 2019;29:262-6.

35. Xie S, Jiang X, Wang R, et al. Low-intensity pulsed ultrasound promotes the proliferation of human bone mesenchymal stem cells by activating PI3K/AKt signaling pathways. J Cell Biochem 2019;120:15823-33.

36. Zhang H, Li ZL, Yang F, et al. Radial shockwave treatment promotes human mesenchymal stem cell self-renewal and enhances cartilage healing. Stem Cell Res Ther 2018;9:54.

37. He R, Chen J, Jiang J, et al. Synergies of accelerating differentiation of bone marrow mesenchymal stem cells induced by low intensity pulsed ultrasound, osteogenic and endothelial inductive agent. Artif Cells Nanomed Biotechnol 2019;47:674-84.

38. Chen J, Jiang J, Wang W, et al. Low intensity pulsed ultrasound promotes the migration of bone marrowderived mesenchymal stem cells via activating FAKERK1/2 signalling pathway. Artif Cells Nanomed Biotechnol 2019;47:3603-13.

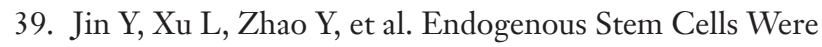
Recruited by Defocused Low-Energy Shock Wave in TreatingDiabeticBladder Dysfunction. Stem Cell Rev Rep 2017;13:287-98.

40. Hochstrasser T, Frank HG, Schmitz C. Dose-dependent and cell type-specific cell death and proliferation following in vitro exposure to radial extracorporeal shock waves. Sci Rep 2016;6:30637.

41. Zhang J, Kang N, Yu X, et al. Radial Extracorporeal Shock Wave Therapy Enhances the Proliferation and Differentiation of Neural Stem Cells by Notch, PI3K/AKT, and Wnt/beta-catenin Signaling. Sci Rep 2017;7:15321.

42. Zaher W, Harkness L, Jafari A, et al. An update of human bone mesenchymal stem cell biology and their clinical uses. Arch Toxicol 2014;88:1069-82.

43. Wei FY, Leung KS, Ii G, et al. Low intensity pulsed ultrasound enhanced mesenchymal stem cell recruitment through stromal derived factor 1 signaling in fracture 
healing. PLoS One 2014;9:e106722.

44. Sansone V, Brañes M, Romeo P. A novel bimodal approach for treating atrophic bone non-unions with extracorporeal shockwave sand autologous mesenchymal stem cell transplant. Med Hypotheses 2018;111:4-7.

45. Lu T, Huang YX, Zhang C, et al. Effect of pulsed electromagnetic field therapy on the osteogenic and adipogenic differentiation of bone marrow mesenchymal stem cells. Genet Mol Res 2015;14:11535-42.

46. Wu Y, Xia L, Zhou Y, et al. Icariin induces osteogenic differentiation of bone mesenchymal stem cells in a MAPK-dependent manner. Cell Prolif 2015;48:375-84.

47. Ning GZ, Song WY, Xu H, et al. Bone marrow mesenchymal stem cells stimulated with low-intensity pulsed ultrasound: Better choice of transplantation treatment for spinal cord injury: Treatment for SCI by LIPUS-BMSCs transplantation. CNS Neurosci Ther 2019;25:496-508.

48. Wang G, Zhang Q, Zhuo Z, et al. Enhanced Homing of CXCR-4 Modified Bone Marrow-Derived Mesenchymal Stem Cells to Acute Kidney Injury Tissues by MicroBubble-Mediated Ultrasound Exposure. Ultrasound Med Biol 2016;42:539-48.

49. Chang X, Liu J, Liao X, et al. Ultrasound-mediated microbubble destruction enhances the therapeutic effect of intracoronary transplantation of bone marrow stem cells on myocardial infarction. Int J Clin Exp Pathol 2015;8:2221-34.

50. Shan HT, Zhang HB, Chen WT, et al. Combination of low-energy shock-wave therapy and bone marrow mesenchymal stem cell transplantation to improve the erectile function of diabetic rats. Asian J Androl 2017;19:26-33.

51. Jiang T, Xu T, Gu F, et al. Osteogenic effect of low intensity pulsed ultrasound on rat adipose-derived stem cells in vitro. J Huazhong Univ Sci Technolog Med Sci 2012;32:75-81.

52. Zhang H, Zhao Y, Wang M, et al. A promising therapeutic option for diabetic bladder dysfunction: Adipose tissuederived stem cells pretreated by defocused low-energy shock wave. J Tissue Eng Regen Med 2019;13:986-96.

53. Poh PSP, Seeliger C, Unger M, et al. Osteogenic Effect and Cell Signaling Activation of Extremely LowFrequency Pulsed Electromagnetic Fields in AdiposeDerived Mesenchymal Stromal Cells. Stem Cells Int 2018;2018:5402853.

54. Nasb M, Liangjiang H, Gong C, et al. Human adiposederived Mesenchymal stem cells, low-intensity pulsed ultrasound, or their combination for the treatment of knee osteoarthritis: study protocol for a first-in-man randomized controlled trial. BMC Musculoskelet Disord 2020;21:33.

55. Wang X, Liu C, Xu Y, et al. Combination of mesenchymal stem cell injection with icariin for the treatment of diabetes-associated erectile dysfunction. PLoS One 2017; 12:e0174145.

56. Ling L, Feng X, Wei T, et al. Effects of low-intensity pulsed ultrasound (LIPUS)-pretreated human amnion-derived mesenchymal stem cell (hAD-MSC) transplantation on primary ovarian insufficiency in rats. Stem Cell Res Ther 2017;8:283.

57. Xia B, Zou Y, Xu Z, et al. Gene expression profiling analysis of the effects of low-intensity pulsed ultrasound on induced pluripotent stem cell-derived neural crest stem cells. Biotechnol Appl Biochem 2017;64:927-37.

58. Yang B, Li M, Lei H. Low Intensity Pulsed Ultrasound Influences the Myogenic Differentiation of Muscle Satellite Cells in a Stress Urinary Incontinence Rat Model. Urology 2019;123:297.e1-297.e8.

59. Wu AK, Zhang X, Wang J, et al. Treatment of stress urinary incontinence with low-intensity extracorporeal shock wave therapy in a vaginal balloon dilation induced rat model. Transl Androl Urol 2018;7:S7-16.

60. Kang N, Peng D, Wang B, et al. The effects of microenergy acoustic pulses on animal model of obesityassociated stress urinary incontinence. Part2: In situ activation of pelvic floor and urethral striated muscle progenitor cells. Neurourol Urodyn 2019;38:2140-50.

61. Behr-Roussel D, Giuliano F. Low-energy shock wave therapy ameliorates erectile dysfunction in a pelvic neurovascular injuries rat model. Transl Androl Urol 2016;5:977-9.

62. Peng D, Yuan H, Liu T, et al. Smooth Muscle Differentiation of Penile Stem/Progenitor Cells Induced by Microenergy Acoustic Pulses In Vitro. J Sex Med 2019;16:1874-84.

63. Lin G, Reed-Maldonado AB, Wang B, et al. In Situ Activation of Penile Progenitor Cells With LowIntensity Extracorporeal Shockwave Therapy. J Sex Med 2017;14:493-501.

64. Lu Z, Lin G, Reed-Maldonado A, et al. Low-intensity Extracorporeal Shock Wave T reatment Improves Erectile Function: A Systematic Review and Meta-analysis. Eur Urol 2017;71:223-33.

65. Hei WH, Byun SH, Kim JS, et al. Effects of electromagnetic field (PEMF) exposure at different 
frequency and duration on the peripheral nerve regeneration: in vitro and in vivo study. Int $\mathrm{J}$ Neurosci 2016;126:739-48.

66. Vincenzi F, Ravani A, Pasquini S, et al. Pulsed electromagnetic field exposure reduces hypoxia and inflammation damage in Neuron-Like and microglial cells. J Cell Physiol 2017;232:1200.

67. Huang C, Holfeld J, Schaden W, et al. Mechanotherapy: revisiting physical therapy and recruiting mechanobiology for a new era in medicine. Trends Mol Med 2013;19:555-64.

68. Takahashi T, Nakagawa K, Tada S, et al. Low-energy shock waves evoke intracellular $\mathrm{Ca} 2$ + increases independently of sonoporation. Sci Rep 2019;9:3218.

69. Yoon CW, Jung H, Goo K, et al. Low-Intensity Ultrasound Modulates Ca2 + Dynamics in Human Mesenchymal Stem Cells via Connexin 43 Hemichannel. Ann Biomed Eng 2018;46:48-59.

70. Gao Q, Cooper PR, Walmsley AD, et al. Role of Piezo Channels in Ultrasound-stimulated Dental Stem Cells. J Endod 2017;43:1130-6.

71. Zhao Q, Zhou H, Chi S, et al. Structure and mechanogating mechanism of the Piezol channel. Nature 2018;554:487-92.

72. Walton TA, Idigo CA, Herrera N, et al. MscL: channeling membrane tension. Pflugers Arch 2015;467:15-25.

73. Puts R, Rikeit P, Ruschke K, et al. Functional regulation of YAP mechanosensitive transcriptional coactivator by Focused Low-Intensity Pulsed Ultrasound (FLIPUS) enhances proliferation of murine mesenchymal precursors. PLoS One 2018;13:e0206041.

74. Pilla AA. Nonthermal electromagnetic fields:from first messenger to therapeutic applications. Electromagn Biol Med 2013;32:123-36.

75. Zhang N, Chow SK, Leung KS, et al. Ultrasound as a stimulus for musculoskeletal disorders. J Orthop Translat 2017;9:52-9.

76. Hatanaka K, Ito K, Shindo T, et al. Molecular mechanisms of the angiogenic effects of low-energy shock wave therapy: roles of mechanotransduction. Am J Physiol Cell Physiol 2016;311:C378-85.

77. Neuman M, Sosnovski V, Kais M, et al. Transobturator vs single-incision suburethral mini-slings for treatment of female stress urinary incontinence:early postoperative pain and 3-year followup. J Minim Invasive Gynecol 2011;18:769-73.

78. Pérez LM, Webster GD. Successful outcome of artificial urinary sphincters in men with post-prostatectomy urinary incontinence despite adverse implantation features. J Urol 1992;148:1166-70.

79. Wang Y, Hadley HR. Experiences with the artificial urinary sphincter in the irradiated patient. J Urol 1992;147:612-3.

80. Vitillo L, Kimber SJ. Integrin and FAK Regulation of Human Pluripotent Stem Cells. Curr Stem Cell Rep 2017;3:358-65.

81. Keren A, Tamir Y, Bengal E. The p38 MAPK signaling pathway: a major regulator of skeletal muscle development. Mol Cell Endocrinol 2006;252:224-30.

82. Lluís F, Ballestar E, Suelves M, et al. E47 phosphorylation by $\mathrm{p} 38$ MAPK promotes MyoD/E47 association and muscle-specific gene transcription. EMBO J 2005;24:974-84.

83. Yu JS, Cui W. Proliferation, survival and metabolism: the role of PI3K/AKT/mTOR signaling in pluripotency and cell fate determination. Development 2016;143:3050-60.

84. Rodgers JT, King KY, Brett JO, et al. mTORC1 controls the adaptive transition of quiescent stem cells from G0 to G(Alert). Nature 2014;510:393-6.

85. Zhou JY, Huang DG, Qin YC, et al. mTORC1 signaling activation increases intestinal stem cell activity and promotes epithelial cell proliferation. J Cell Physiol 2019;234:19028-38.

86. Zou Z, Chen J, Liu A, et al. mTORC2 promotes cell survival through c-Myc-dependent up-regulation of E2F1. J Cell Biol 2015;211:105-22.

87. Liu T, Shindel AW, Lin G, et al. Cellular signaling pathways modulated by low-intensity extracorporeal shock wave therapy. Int J Impot Res 2019;31:170-6.

88. López-Marín LM, Rivera AL, Fernandez F, et al. Shock wave induced permeabilization of mammalian cells. Phys Life Rev 2018;26-27:1-38.

89. Li Y, Lee C, Chen R, et al. A feasibility study of applications of single beam acoustic tweezers. Appl Phys Lett 2014;105:173701.

Cite this article as: Chen Y, Cai Q, Pan J, Zhang D, Wang J, Guan R, Tian W, Lei H, Niu Y, Guo Y, Quan C, Xin Z. Role and mechanism of micro-energy treatment in regenerative medicine. Transl Androl Urol 2020;9(2):690-701. doi: 10.21037/ tau.2020.02.25 